\title{
Valoración de las familias participantes en el programa preventivo Crecer en Familia con Gorgoritos
}

Alicia Benavides Nieto. Universidad de Granada

Marta Molina Bernal. Universidad de Granada

María Fernández Cabezas. Universidad de Granada

Recepción: 10.02.2019 | Aceptado: 15.10.2019

Correspondencia a través de ORCID: Alicia Benavides

iD $0000-0002-1337-412 X$

Citar: Benavides, A., Molina, M. y Fernández, M. (2019). Valoración de las familias participantes en el programa preventivo Crecer en Familia con Gorgoritos. ReiDoCrea, 8, 202-211.

Resumen: Antecedentes: Resulta de vital importancia apoyar a las familias en la crianza de los menores en las primeras etapas para evitar posibles problemas de convivencia y de conducta. Para responder a esta necesidad, se ha implementado el programa preventivo de intervención Crecer en Familia con Gorgoritos con familias que tienen hijos e hijas en edad infantil. Objetivos: Conocer la satisfacción de las familias participantes en Crecer en Familia con Gorgoritos, comparar los resultados de las valoraciones obtenidas en dos cursos diferentes entre los años 2016 y 2018 y proponer mejoras para futuras implementaciones. Método: Evaluamos la participación de 14 familias (9 madres y 5 padres) a través de un diseño mixto, utilizando un cuestionario ad hoc y entrevistas semiestructuradas. Resultados: Las familias valoran positivamente el programa exceptuando la frecuencia y duración de este, al igual que el contenido trabajado en el escaso tiempo realizado, siendo éstas las variables peor valoradas. Conclusiones: Resulta beneficioso para las familias el que existan programas dirigidas a ellas por la gran influencia que ejercen en el desarrollo de los menores y en la funcionalidad familiar desde edades tempranas.

Palabras clave: Papel de la Familia | Medio familiar

Assessment of the participating families in the Crecer en Familia con Gorgoritos preventive program

\begin{abstract}
Antecedents: It is essential to support families in the upbringing of children in the early stages in order to avoid possible coexistence and behavior problems. In order to respond to this need, the preventive program Crecer en Familia con Gorgoritos has been implemented with families who have children at preschool age. Objectives: To know the opinion of the participating families in Crecer en Familia con Gorgoritos, to compare the results of the evaluations obtained in two different years between 2016 and 2018 and to propose improvements for future implementations. Method: We evaluated the participation of 14 families (9 mothers and 5 fathers) through a mixed design, using an ad hoc questionnaire and semistructured interviews. Results: The families value the program positively except for its frequency and duration, as well as the content worked in the short time, these being the worst valued variables. Conclusions: It is beneficial for families that there are programs aimed at them because of the great influence they have on the development of children and on family functionality from an early age.
\end{abstract}

Keywords: Family Role | Family Environment

\section{Introducción}

Debido a los diversos cambios producidos en la humanidad, como son la incorporación de la mujer al mercado laboral y la evolución tecnológica que influye en el día a día de las relaciones familiares, resulta muy compleja la función que deben desempeñar las madres y padres como agentes de socialización para sus hijos e hijas. Actualmente los estilos o funciones ejercidas por los tutores tienden a reforzar la sobreprotección y disminuir los límites y las normas como medida de negociación (Gil, 2007), lo que puede derivarse en un problema conductual del menor y a su vez en problemas de convivencia familiar.

Teniendo en cuenta lo mencionado por autores como Solís-Cámara, Medina y Díaz (2014), las madres y los padres pueden actuar como agentes de protección o agentes de riesgo, lo que supone que según cómo actúen les influirá de un modo positivo o 
negativo a sus hijas/os, aumentando o reduciendo la aparición de conductas prosociales, entendiéndose como el "conjunto de comportamientos humanos voluntarios a favor de otros seres humanos, con independencia de que en muchos casos puede revertir en beneficios propios" (Urquiza y Casullo, 2006, p. 298). Entendemos como factor de protección los que resultan adecuados para el desarrollo positivo del o la menor, promoviendo, por ejemplo, un rol paterno positivo y teniendo un manejo adecuado del coraje también denominado autocontrol. Por otro lado, los factores de riesgo son aquellos aspectos que influyen negativamente en el correcto desarrollo social del o la menor, como pueden ser la disciplina severa y el estrés. A su vez, Ramirez (2002) afirma que las prácticas y estilos de crianza que emplean las familias están relacionados con la aparición de problemas de conducta en los menores. Por tanto, resulta necesario realizar programas de intervención tanto con menores, para disminuir las conductas disruptivas desde edades tempranas, como con las familias, por ser una figura muy importante en su desarrollo. A este respecto, contando con estudios previos que afirman la efectividad de realizar programas de prevención, y teniendo en cuenta los distintos niveles de prevención según Gordon (1987,citado en Justicia-Arraez, 2014), siendo el nivel primario o universal, secundario o selectiva y terciario o indicada, el primer nivel de prevención estaría destinado a todas aquellas intervenciones donde se trabaja con una población general, la cual no presenta riesgos aparentes de padecer el problema por el que se quiere realizar la intervención. En cambio, en el segundo nivel se intervendría con una población que tenga riesgo de padecer el problema por el que se quiere intervenir. $Y$ finalmente el último nivel es de prevención terciario o indicado dirigido a una población que ya está afectada o presenta indicios de tener el problema por el que se quiere intervenir para mejorarlo o solucionarlo. Entre ellos, el nivel más efectivo es el nivel de prevención primaria (Quintero-Fleites et al. 2017), siendo el que se realiza antes de la aparición de la problemática en una población que aparentemente no tiene el riesgo de padecerla, de tal modo que las personas normalizan e integran mejor el aprendizaje y los valores trasmitidos en la intervención que pretende evitar la aparición del problema.

Para tratar de responder a dichas cuestiones existen algunos programas enfocados a establecer medidas de prevención del comportamiento antisocial en menores desde la etapa infantil hasta su adolescencia partiendo de la modificación de la dinámica familiar, como Parent-Child Interaction Therapy (Hembree-Kigin y McNeil, 1995) e Incredible Years (Webster-Stratton, 2001). Ambos programas pretenden mejorar la relación familiar considerando las emociones y las competencias sociales como base fundamental en la interacción. Del mismo modo, Crecer en Familia con Gorgoritos, el programa que se desarrolla en el presente estudio, trata de responder a las necesidades que tienen las familias facilitándoles información sobre las temáticas que ellas consideran necesarias para un mejor ambiente familiar y el comportamiento de sus hijas e hijos.

Con el fin de poder trabajar diferentes aspectos y ámbitos de desarrollo se han abordado las temáticas propuestas en cuatro bloques. Las temáticas tratadas han sido distribuidas en los bloques según las necesidades e intereses que presentaban las madres y padres participantes del programa. Se han mantenido los cuatro bloques en los dos cursos escolares evaluados en la presente investigación, aunque ha habido variaciones de las temáticas (ver tabla 1).El primer bloque trata los sentimientos y las emociones, el segundo, las habilidades comunicativas, el tercero la importancia de establecer normas y límites de forma adecuada, y en el cuarto bloque trabajamos la autonomía, promoviendo la ayuda y la cooperación de los menores atendiendo a sus distintos niveles de desarrollo. 
Tabla 1. Bloques y temáticas del programa Crecer en Familia con Gorgoritos.

\begin{tabular}{|c|c|c|c|c|}
\hline Bloques & & Temáticas curso 2016/17 & & Temáticas curso 2017/18 \\
\hline $\begin{array}{l}\text { BLOQUE 1: } \\
\text { Sentimientos y } \\
\text { emociones }\end{array}$ & 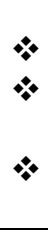 & $\begin{array}{l}\text { Sesión 1: Madres perfectas. } \\
\text { Sesión 2: Relajación y } \\
\text { emociones. } \\
\text { Sesión 3: Desarrollo } \\
\text { evolutivo y rabietas. }\end{array}$ & $\%$ & $\begin{array}{l}\text { Sesión 1: Madres perfectas. } \\
\text { Sesión 2: Relajación y } \\
\text { emociones. } \\
\text { Sesión 3: Emociones y } \\
\text { rabietas. }\end{array}$ \\
\hline $\begin{array}{l}\text { BLOQUE 2: } \\
\text { Habilidades } \\
\text { comunicativas }\end{array}$ & $*$ & $\begin{array}{l}\text { Sesión 5: Estilos educativos } \\
\text { parentales. } \\
\text { Sesión 6: Acoso escolar. }\end{array}$ & 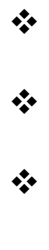 & $\begin{array}{l}\text { Sesión 4: Rabietas ¿Cómo } \\
\text { prevenirlas? } \\
\text { Sesión 7: Conflictos entre } \\
\text { hermanos. } \\
\text { Sesión 9: Ciclo de conductas } \\
\text { desadaptativas. }\end{array}$ \\
\hline $\begin{array}{l}\text { BLOQUE 3: } \\
\text { Establecimiento } \\
\text { de límites y } \\
\text { normas }\end{array}$ & $*$ & $\begin{array}{l}\text { Sesión 4: Rabietas, y normas } \\
\text { y límites. }\end{array}$ & $*$ & $\begin{array}{l}\text { Sesión 5: Casos prácticos } \\
\text { ¿Cómo prevenir las rabietas? } \\
\text { Sesión 6: Estilos parentales. }\end{array}$ \\
\hline $\begin{array}{l}\text { BLOQUE 4: } \\
\text { Ayuda y } \\
\text { cooperación } \\
\text { promoviendo la } \\
\text { autonomía }\end{array}$ & $*$ & $\begin{array}{l}\text { Sesión 7: Independencia, } \\
\text { autonomía y seguridad. } \\
\text { Sesión 8: Importancia del } \\
\text { juego. }\end{array}$ & $*$ & $\begin{array}{l}\text { Sesión 8: Independencia, } \\
\text { autonomía y seguridad. } \\
\text { Sesión 10: Importancia de la } \\
\text { familia. } \\
\text { Sesión 11: Importancia del } \\
\text { juego. }\end{array}$ \\
\hline
\end{tabular}

Como muestra la tabla anterior, los bloques presentes en el programa se encuentran abiertos y son flexibles ya que muchos de los temas tratados en las sesiones se trabajan de forma transversal en el contenido de varios bloques. La elección de los bloques parten de la importancia de promover el aumento de los factores de protección y reducir los factores de riesgo comentados con anterioridad, además de tener en cuenta las tres funciones básicas que cumplen las familias (Fernández De Haro, 1996). Estas funciones son la trasmisión de valores a la persona, la conservación de la coherencia entre el propio miembro de la familia, la familia y la sociedad, y la enseñanza y aplicación de las normas sociales para vivir en sociedad.

Dentro de cada una de las sesiones se han realizado diferentes actividades plasmadas en las tablas 3 y 4 (anexos). Las sesiones han tenido una duración de 45 minutos y se realizaban cada dos/tres semanas, mientras los infantiles recibían clase de música, los familiares podían asistir a las sesiones del programa.

Tras esta breve exposición acerca del programa Crecer en Familia con Gorgoritos, y qué temáticas se trabajan en el mismo, en la presente investigación se pretende conocer la valoración de las familias participantes en el programa y comparar los resultados de las valoraciones obtenidas en los grupos en dos cursos académicos: 2016/17 y 2017/18. Asimismo, se pretende evaluar las posibles mejoras de cara a incorporarlas en futuras implementaciones del programa.

\section{Método}

Atendiendo a las características de nuestra investigación, la metodología empleada presenta un diseño mixto, es decir, hemos recogido datos de corte cuantitativos como cualitativos (Bisquerra, 2016).Concretamente hemos empleado cuestionarios con 
preguntas tipo Likert y abiertas, así como entrevistas de corte cualitativo, para conocer con más profundidad sus opiniones acerca de la participación en el programa. Este tipo de diseño resulta muy beneficioso cuando se pretende recoger información de forma sistemática y a la vez trata de conocer la percepción de los participantes del programa desde una perspectiva más cercana y holística.

\section{Participantes}

Las familias participantes en el programa fueron un total de 30 en ambos cursos académicos (12 en el curso 2016/17 y 18 en el curso 2017/18). No obstante, la muestra recogida para los cuestionarios de satisfacción consta de 14 familiares que asistieron a más del $70 \%$ de las sesiones propuestas. Las edades de los mismos oscilan entre los 31 y 48 años de edad, siendo en su mayoría mujeres ( 9 mujeres y 5 hombres), y todos coincidían en tener en su unidad familia la presencia de un menor de entre 0 a 6 años. Por otra parte, el número de entrevistados fueron 5 familiares, empleando para ello un muestreo no probabilístico de tipo causal (Bisquerra, 2016).

Todos los asistentes eran madres o padres de niños y niñas en edad infantil, quienes estaban interesados en conocer nuevos aspectos, perspectivas, pautas y herramientas para promover un adecuado funcionamiento familiar.

Resulta conveniente mencionar que de los 14 encuestados, 6 de ellos son familiares del grupo del curso académico 2016/17, y los 8 restantes son familiares del curso 2017/18.

\section{Instrumentos}

Para la presente investigación se ha recogido información a través de un cuestionario y entrevistas semiestructuradas con el objetivo de conocer la opinión de las familias participantes sobre el programa una vez finalizado.

El cuestionario sobre la valoración del programa es ad hoc, es decir, se ha elaborado para evaluar el presente programa, y presenta una escala tipo likert de 1 ("Totalmente en desacuerdo") a 7 ("Totalmente de acuerdo"). Estudia siete dimensiones, entre ellas la importancia trabajar aspectos educativos con las familias (ítems 1 y 2 ), actividades y temas trabajados en el proyecto (ítems 3, 5, 8, 10 y el ítem 23 invertido), la participación y cambio personal en las diferentes sesiones (ítems $6,12,13,14,15,16,17,18$ y 19), la duración (ítem 7 y los ítems 21 y 22 invertidos), las habilidades mostradas en las monitoras (ítems 11), la metodología empleada en el programa (ítems 20 y 24 invertidos), y la satisfacción enlas sesiones (ítems 4, 9 y 25). La presencia de ítems invertidos o reverse score se deben tener en cuenta para analizar los resultados obtenidos de una forma adecuada, ya que generalmente presentan una connotación contraria a la que se estudia, es decir, la pregunta se realiza en forma negativa cuando se estudia la forma afirmativa de la dimensión.

El cuestionario empleado consta de fiabilidad y validez aceptable $(\alpha=.664)$. Es válido al proceder de una adaptación de un cuestionario validado anteriormente por juicio de expertos, y es fiable porque en el estudio de alfa de Cronbach realizado en este análisis es cercano a $\alpha=.7$, siendo una medida aceptable según George y Mallery (2003).

Las entrevistas utilizadas son semiestructuradas. Este tipo de entrevistas se caracterizan por aportar más información que una entrevista cerrada o estructurada, por tanto, es un tipo de entrevista abierta que permite, dentro de las preguntas previamente realizadas, que la entrevistada/o pueda extenderse en su explicación y así dar más detalles o aportar mayor información al respecto (Bisquerra, 2016). 


\section{Procedimiento}

Previo a la implementación del programa, se informó a las familias participantes en las clases de música de Gorgoritos. Mientras que sus hijos e hijas asisten a clases de música, las familias pueden asistir a las sesiones de Crecer en Familia con Gorgoritos.

En la primera sesión se recogió información sobre las necesidades o temáticas que madres y padres querían tratar, de esta forma se planificaron las sesiones conforme a los intereses de las familias ubicándolas en los cuatro bloques mencionados.

Al finalizar las sesiones (en el curso 2016/17 se realizaron 8 sesiones y en el curso $2017 / 18$ se hicieron 11) se les entregó el cuestionario sobre la valoración del programa para conocer su opinión sobre el programa y las posibles sugerencias en la implementación futura. Asimismo, se les propuso realizar una entrevista a quienes quisieran aportar más información sobre su opinión con respecto a su experiencia en la participación del programa.

\section{Análisis de datos}

Tras la recogida de datos, procedemos a analizar la información obtenida del cuestionario empleando el paquete SPSS v. 22 y Excel. Se han realizado análisis descriptivos, de frecuencias y correlaciones. Para la parte cualitativa, se procede a realizar un análisis de contenido de las entrevistas realizadas, que servirán como complemento a los resultados obtenidos en el cuestionario.

\section{Resultados}

Como puede apreciarse en la Figura 1, todos los resultados obtenidos procedentes del cuestionario se encuentran cercanos a la puntuación 7 , traduciéndose en "totalmente de acuerdo", lo que indica que en la mayoría de las familias participantes muestran la una elevada satisfacción por el programa. No obstante, las variables Frecuencia/Duración (4.14) y Contenido (4.89) son las que presentan puntuaciones más bajas, lo que indica que ambos aspectos son los que los familiares consideran que deben modificarse en el desarrollo de las sesiones.

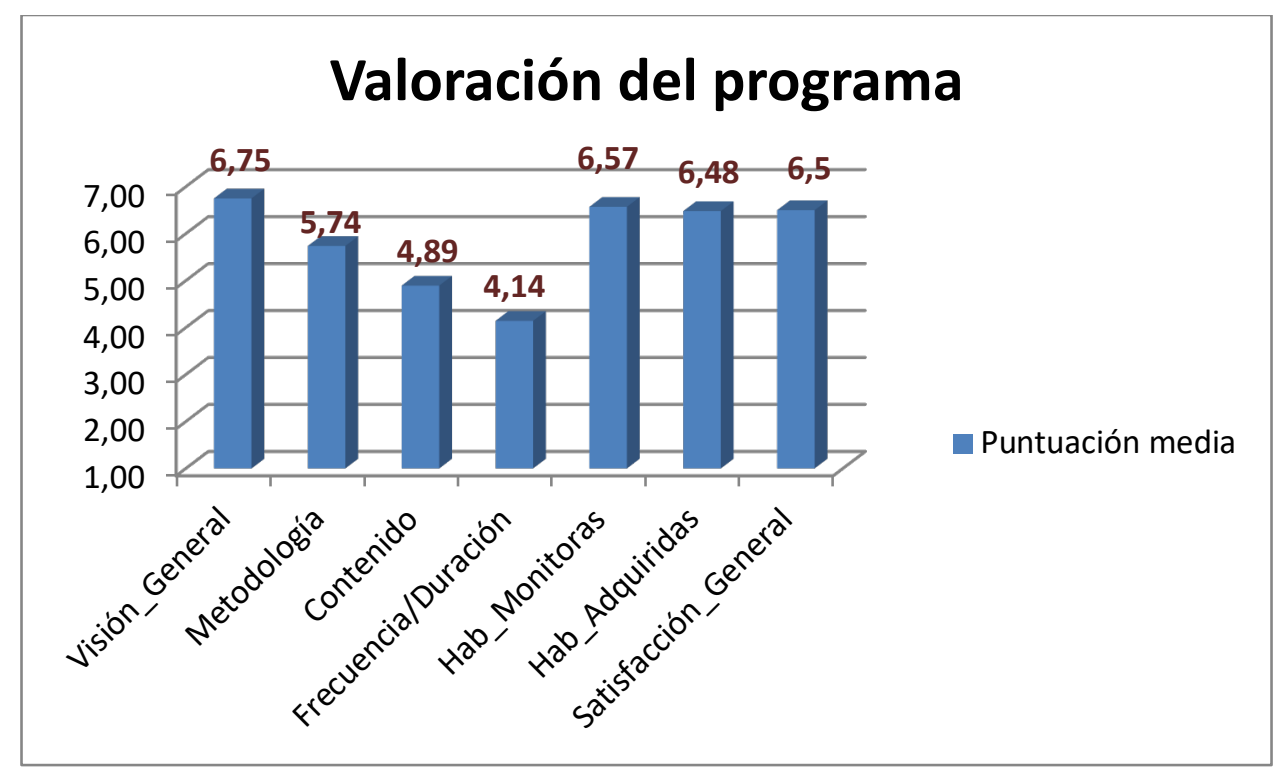

Figura 1. Puntuación media obtenida de las variables estudiadas $(n=14)$. 
Atendiendo a los comentarios recibidos a través de las entrevistas, se puede observar que ambas variables se encuentran relacionadas cuando se les preguntaba por alguna propuesta de mejora al programa:

"Si podéis hacer el programa con las sesiones un poco más largas pues... a lo mejor sería conveniente, que es difícil porque aprovecháis el tiempo de las clases. Pero realmente es bastante interesante, además en algunos temarios hace falta profundizar un poquito más. (E1)"

"Mmmm... no sé en todo caso, quizás, a lo mejor algunos enlaces, y más material para luego seguir mirando. (E2)"

Por otro lado, las variables con puntuaciones más altas son la Visión general (6.75), Habilidades de las monitoras (6.57), Satisfacción general (6.5) y Habilidades adquiridas (6.48). Lo que indica que las familias consideran que este tipo de programas son muy importantes para el desarrollo de las personas, que las monitoras que impartían el programa tenían las habilidades necesarias para desarrollarlo, la satisfacción tras haber asistido al programa era positiva, y que han aprendido diversas habilidades tras asistir a las sesiones. En cuanto a las Habilidades adquiridas son mencionadas varias veces por las entrevistadas y entrevistados al responder a preguntas como cuál ha sido su experiencia en la participación en el programa, o en qué medida ha cambiado su dinámica familiar:

"Pues me viene bien para ampliar mis recursos como madre para poder afrontar situaciones que sin haber tenido la experiencia de que pasa, "a viva voz", lo que pasan otras madres y otros padres, realmente me ha venido genial para reforzar mis recursos, para saber cómo afrontar otras situaciones determinadas en la que necesito mantener la calma. Y también me ha venido bien, tanto como juego, para poder jugar con mi hija como para poder enseñarle pedagógicamente mejor... (E3)"

"En vez de por ejemplo prohibirle que haga algo darle una alternativa de lo que si puede hacer ¿no? Eso sí que me he esforzado. Por ejemplo, pues en vez de decirle no hagas ruido, ¿por qué no jugamos a esto que es más tranquilo? ¿No? Ese tipo de cosas por ejemplo me he esforzado mucho en intentar conseguirlo. Todavía...estoy en ello, y sigo (se ríe) porque esto no es, no es fácil...(E4)"

Por otro lado, y considerando las diferencias obtenidas entre las medias atendiendo al curso académico en el que se ha implementado el programa Crecer en Familia con Gorgoritos, se puede observar en la Tabla 2 que los participantes del curso 2016/17 presentan medias más altas que los participantes en el curso 2017/18 en la mayoría de las escalas estudiadas, exceptuando la de Habilidades adquiridas. Es decir, a pesar de tener menor satisfacción por la asistencia al programa, las familias asistentes en el curso 2017/18 consideran haber adquirido habilidades que les han sido útiles a la hora de relacionarse con sus pequeños (6.7083 sobre 7 ), valorando con mayor puntuación que los asistentes en el curso 2016/17 (6.1852). Las diferencias obtenidas en ambos grupos con respecto a las Habilidades adquiridas presentan diferencias estadísticamente significativas $(p=0.05)$, lo que implica que estas diferencias en la puntuación no se deben al azar. En cambio, las diferencias obtenidas entre la satisfacción de ambos grupos no presentan diferencias estadísticamente significativas $(p=0.767)$, y por lo tanto puedan deberse al azar. 
Tabla 2. Puntuaciones medias de cada curso (n curso 2016/17=6; n curso 2017/18=8).

\begin{tabular}{lcc}
\hline Variables del cuestionario & Curso 2016/17 & Curso 2017/18 \\
\hline Visión general & 6.8333 & 6.6875 \\
\hline Metodología & 5.7778 & 5.7083 \\
\hline Contenido & 4.9167 & 4.8750 \\
\hline Frecuencia/ Duración & 5.1667 & 3.3750 \\
\hline Habilidades de las monitoras & 6.8333 & 6.3750 \\
\hline Habilidades adquiridas & & \\
\hline & 6.1852 & 6.7083 \\
\hline Satisfacción general & 6.6667 & 6.3750 \\
\hline
\end{tabular}

Otro de los resultados obtenidos en cuanto a las posibles correlaciones de las variables estudiadas en el cuestionario, muestra que existen correlaciones en las variables Visión general y Metodología empleada en el programa, Visión y Satisfacción generales, en Metodología y Contenido, y en Satisfacción general y Metodología utilizada en las sesiones. Todas estas correlaciones son positivas, es decir, si una de las variables aumenta, la otra aumenta también por lo que, si por ejemplo la Satisfacción general es puntuada de forma elevada, la valoración sobre la Metodología empleada en las sesiones también será mayor, y viceversa.

\section{Discusión}

La crianza familiar puede resultar una tarea muy complicada, porque no todas las familias son iguales, ni por supuesto todos los infantiles lo son. Considerando este punto y el hecho de los escasos espacios de formación y encuentro para las familias sobre cómo afrontar ciertas situaciones que les preocupan en relación con sus hijos e hijas, se deben poner en práctica intervenciones que respondan a dicha necesidad social y familiar. Como mencionan Nelsen, Erwin, y Duffy (2014, p.37) las madres y los padres "deben aprender a conocer bien a sus hijos y decidir qué es lo más importante en sus vidas", pero siempre conviene tener algún apoyo al respecto para saber cómo actuar o qué estrategias son más apropiadas a emplear en distintos momentos o etapas de la crianza.

En este estudio se ha evaluado la valoración de las familias participantes en el programa Crecer en Familia con Gorgoritos: programa de prevención primaria o universal. Concretamente el programa Crecer en Familia con Gorgoritos ha obtenido una alta valoración por parte de las familias que han recibido la formación de este. Los resultados arrojados indican que los familiares se sienten más capacitados para establecer una relación más segura con sus hijos e hijas, y saben que compartir su experiencia con 
otras personas que se encuentran en la misma situación es muy positivo porque pueden encontrar apoyo y respuestas a sus dudas. Asimismo, reconocen que la crianza de los infantiles supone un continuo esfuerzo en todas sus etapas de desarrollo. Además, para continuar con ese proceso de enseñanza-aprendizaje se debe tener paciencia y mantener la calma debido a que no es una tarea sencilla.

No obstante, habría que mejorar la frecuencia (una vez por semana, por ejemplo) y duración (más de 45 minutos) de las sesiones para así poder aumentar los contenidos, así como tratar con mayor profundidad las temáticas. Se ha comprobado que las familias que han recibido un mayor número de sesiones sienten haber aumentado en mayor medida las habilidades adquiridas con respecto a ejercer su función materna o paterna con los pequeños de la familia. Esto se puede traducir en una mejora en la dinámica familiar, buena relación padre/madre-hijo/hija, mejor comportamiento del menor y de los progenitores hacia ellos/as.

También se han encontrado diferencias en cuanto a la satisfacción del programa, concretamente a mayor número de sesiones menor satisfacción hacia el programa. Esto puede deberse a la incorporación tardía de algunos familiares al programa en el grupo del último curso. Aunque estos resultados no presentan diferencias estadísticamente significativas y por lo tanto puedan deberse al azar, podríamos emplear los resultados obtenidos en las correlaciones realizadas para, en futuras sesiones, prestar mayor atención a la metodología empleada en las actividades y sesiones realizadas de cara a mejorar la satisfacción: de "muy de acuerdo" a "totalmente de acuerdo". En este sentido, la escasa frecuencia (cada dos/tres semanas) o duración (45 minutos) del programa repercute a la falta de profundización en los contenidos tratados en las sesiones, a no ser que como bien indica la Entrevitada2 (E2) se facilite más material para que revisen en casa de cara a profundizar en las temáticas que tratamos en las sesiones.

Resumiendo, la metodología se encuentra muy ligada a los contenidos tratados en el programa, por lo que en futuras implementaciones del programa deberíamos considerar tanto la ampliación de contenido como la profundización de este, además de la metodología a empelar.

Todas las personas pasamos por diferentes fases en nuestras vidas y en cada una de ellas necesitamos diferentes estímulos o estrategias para poder paliar las diferentes necesidades y circunstancias que tenemos en cada momento, pero sin duda, el período más significativo para toda persona es la infancia. Robles y Romero (2011) consideran que la intervención temprana es la más eficaz para trabajar los posibles malos hábitos y problemas de comportamiento infantiles, así como para fomentar prácticas y conductas adecuadas en las personas. Así mismo, otros autores como Bronfrenbrenner (1987) y Sameroff, y Chandler (1975) advierten de la influencia que ejercen los distintos sistemas que rodean a los individuos, sobretodo el microsistema siendo el más cercano al menor, traduciéndose este caso como las familias y los parientes más allegados a los menores.

Resaltamos, por ello, la importancia de realizar actividades de formación con las familias como un método efectivo para mejorar las competencias familiares y sociales y el desarrollo seguro de los más pequeños. Pero en la sociedad actual aún sigue siendo una necesidad escasamente cubierta porque son pocas las respuestas que se encuentran desde los organismos públicos, las escuelas o educación no formal desde un enfoque de prevención universal. Además, a esto se añade el escaso tiempo dedicado por parte de las familias a los infantiles tras una larga y cansada jornada laboral o ciertas teorías implícitas acerca de la función familiar. También se debe hacer especial mención a la todavía desigualdad existente en bastantes familias acerca de las tareas 
relativas a la educación de los pequeños, siendo un trabajo mayoritariamente realizado por las madres. Sin ir más lejos, aún siguen siendo más madres que padres las que han asistido a este programa.

Para dar respuesta a estas amenazas y debilidades encontradas a la hora de implementar este tipo de programas, resulta conveniente que además de evaluarlo se divulgue con el fin de poner en conocimiento la importancia de las familias en la educación de los infantiles, ya que la "investigación que no se publica no existe" (Revista Facultad de Ingeniería, 2014).

\section{Referencias}

Abidin, R. R. (1995). Parenting Stress Index (3a ed.). Odessa, FL, EE.UU.: Psychological Assessment Resources. Alonso, J. y Román, J.M. (2003). PEF: escalas de identificación de «prácticas educativas familiares». Madrid: Editorial Cepe.

Bisquerra, R. (2016). Metodología de la investigación educativa (5a ed.). Madrid: La Muralla.

Bronfenbrenner, U. (1987). La ecología del desarrollo humano. Barcelona: Paidós.

Fernández De Haro, E. (1996). La familia como marco comunitario de educación multicultural para la tolerancia (73-90), en J.A. Ortega (coord.) Educación multicultural para la tolerancia y la paz.Granada: GEU.

George, D. y Mallery, P. (2003). Reliability analysis. SPSS for Windows, step by step: a simple guide and reference. Boston: Allyn \& Bacon.

Gil, M. Á. (2007). La familia: claves para una correcta gestión de las personas y situaciones familiares. Madrid: Editorial AMAT.

Hembree-Kigin, T.L. y McNeil, C.B. (1995). Parent-child interaction therapy. New York: Plenum Press.

Justicia-Arráez, A. (2014). Estudio longitudinal de los efectos del programa Aprender a Convivir en Educación Infantil [Tesis Doctoral]. Universidad de Granada, Granada, España.
Nelsen, J., Erwin, C., y Duffy, R.A. (2014). Disciplina positiva para preescolares: educar niños responsables, respetuosos y capaces. Barcelona: Omega.

Ramírez, M. (2002). Prácticas de crianza de riesgo y problemas de conducta en los hijos. Apuntes de Psicología, 20 (2), 273-282.

Revista Facultad de Ingeniería (2014). La importancia de publicar los resultados de Investigación. Revista Facultad de Ingeniería, 37(23), 24-35.

Robles, Z. y Romero, E. (2011). Programas de entrenamiento para padres de niños con problemas de conducta: una revisión de su eficacia. Anales de psicología 27 (1), 86-101.

Sameroff, A. J., y Chandler, M. J. (1975). Reproductive risk and the continuum of caretaking casualty. Review of child development research, 4, 187-244.

Solís-Cámara, Medina, y Díaz (2014). Relaciones entre la crianza y factores protectores o de riesgo, antes y después de una intervención para padres. SUMMA psicológica UST, 11 (1), 7587.

Urquiza, V. y Casullo, M. M. (2006). Empatía, razonamiento moral y conducta prosocial en adolescentes. Anuario de investigaciones, 13, 297-302.

Webster-Stratton, C. (2001). The Increible Years Trainings Series. Office of Juvenile Justice and Delinquency Prevention. Juvenile Justice Bulletin, 1-24. 
Anexos. Esquema de las sesiones y actividades realizadas.

Tabla 3. Esquema de actividades propuestas en las sesiones curso 2016/17.

\begin{tabular}{|c|c|c|}
\hline $\begin{array}{l}\text { SESIÓN 1: } \\
\text { "MADRES PERFECTAS" }\end{array}$ & $\begin{array}{l}> \\
> \\
>\end{array}$ & $\begin{array}{l}\text { Actividad 1: Presentación. } \\
\text { Actividad 2: Visualización del vídeo "La mamá perfecta". } \\
\text { Actividad 3: ¿Por qué soy madre/padre perfecta/o? } \\
\text { Actividad 4: Análisis de necesidades. }\end{array}$ \\
\hline $\begin{array}{c}\text { SESIÓN 2: } \\
\text { "RELAJACIÓN Y EMOCIONES" }\end{array}$ & $>$ & $\begin{array}{l}\text { Actividad 1: Técnicas de relajación. } \\
\text { Actividad 2: Importancia de las emociones. } \\
\text { - } \quad \text { Monstruo de colores. } \\
\text { - Collage de las emociones. }\end{array}$ \\
\hline $\begin{array}{c}\text { SESIÓN 3: } \\
\text { "DESARROLLO EVOLUTIVO Y RABIETAS" }\end{array}$ & $>$ & $\begin{array}{l}\text { Actividad 1: Visualización del video "Para reunión con padre de familia". } \\
\text { Actividad 2: Estrategias para la prevención de rabietas. }\end{array}$ \\
\hline $\begin{array}{c}\text { SESIÓN 4: } \\
\text { "RABIETAS, Y NORMAS Y LÍMITES" }\end{array}$ & $\vec{\lambda}$ & $\begin{array}{l}\text { Actividad 1: Alimentación. } \\
\text { Actividad 2: Estrategias para prevención de rabietas y normas y límites". }\end{array}$ \\
\hline $\begin{array}{c}\text { SESIÓN 5: } \\
\text { "ESTILOS EDUCATIVOS PARENTALES }\end{array}$ & $>$ & $\begin{array}{l}\text { Actividad 1: Detectar estilos parentales en situaciones prácticas. } \\
\text { Actividad 2: Reflexión estilo educativo propio. }\end{array}$ \\
\hline $\begin{array}{c}\text { SESIÓN 6: } \\
\text { "ACOSO ESCOLAR" }\end{array}$ & $>$ & $\begin{array}{l}\text { Actividad 1: Presentación del bullying. } \\
\text { Actividad 2: Protocolo a seguir en caso de acoso escolar. } \\
\text { Actividad 3: Semáforo. }\end{array}$ \\
\hline $\begin{array}{c}\text { SESIÓN 7: } \\
\text { "INDEPENDENCIA, AUTONOMÍA Y SEGURIDAD" }\end{array}$ & $>$ & $\begin{array}{l}\text { Actividad 1: ¿Cómo fomentar en los infantiles la autonomía? } \\
\text { Actividad 2: Los conflictos ¿son positivos o negativos? } \\
\text { Actividad 3: Estrategias y casos prácticos para resolver conflictos. }\end{array}$ \\
\hline $\begin{array}{l}\text { SESIÓN 8: } \\
\text { "IMPORTANCIA DEL JUEGO" }\end{array}$ & $>$ & $\begin{array}{l}\text { Actividad 1: Junglespeed. } \\
\text { Actividad 2: Evaluación del programa. } \\
\text { Actividad 3: Cuento de Resi. }\end{array}$ \\
\hline
\end{tabular}

Tabla 4. Esquema de actividades propuestas en las sesiones curso 2017/18.

\begin{tabular}{|c|c|c|}
\hline $\begin{array}{c}\text { SESIÓN 1: } \\
\text { "MADRES PERFECTAS" }\end{array}$ & $\begin{array}{l}> \\
> \\
>\end{array}$ & $\begin{array}{l}\text { Actividad 1: Actividad de presentación. } \\
\text { Actividad 2: Visualización del vídeo "La mamá perfecta". } \\
\text { Actividad 3: ¿Por qué soy madre/padre perfecta/o? } \\
\text { Actividad 4: Análisis de necesidades. }\end{array}$ \\
\hline $\begin{array}{c}\text { SESIÓN 2: } \\
\text { "RELAJACIÓN Y EMOCIONES" }\end{array}$ & $>$ & $\begin{array}{l}\text { Actividad 1: Técnicas de relajación. } \\
\text { Actividad 2: Introducción hacia las emociones (autocontrol). }\end{array}$ \\
\hline $\begin{array}{c}\text { SESIÓN 3: } \\
\text { "EMOCIONES Y RABIETAS" }\end{array}$ & $>$ & $\begin{array}{l}\text { Actividad 1: Importancia de las emociones } \\
\text { Actividad 2: ¿Qué son las rabietas? }\end{array}$ \\
\hline $\begin{array}{c}\text { SESIÓN 4: } \\
\text { "RABIETAS, ¿CÓMO PREVENIRLAS?" }\end{array}$ & $>$ & Actividad 1: Estrategias para prevención de rabietas y normas y límites. \\
\hline $\begin{array}{l}\text { SESIÓN 5: } \\
\text { "CASOS PRÁCTICOS: ¿CÓMO } \\
\text { PREVENIR LAS RABIETAS?" }\end{array}$ & $\begin{array}{l}> \\
>\end{array}$ & $\begin{array}{l}\text { Actividad 1: Problemas a la hora de ir a dormir ¿Cómo afrontarlos? } \\
\text { Actividad 2: Casos prácticos de rabietas. } \\
\text { Actividad 3: Técnica resolución de conflictos. }\end{array}$ \\
\hline $\begin{array}{c}\text { SESIÓN 6: } \\
\text { "ESTILOS PARENTALES" }\end{array}$ & $>$ & $\begin{array}{l}\text { Actividad 1: Preguntas y presentación del cuadro de los distintos de estilos parentales } \\
\text { Actividad 2: Visualización del siguiente vídeo: } \\
\text { https://www.youtube.com/watch?v=M7qSYKkqS_o } \\
\text { Actividad 3: Importancia de establecer normas y límites, y cómo establecerlas. }\end{array}$ \\
\hline $\begin{array}{c}\text { SESIÓN 7: } \\
\text { "CONFLICTOS ENTRE HERMANOS" }\end{array}$ & $>$ & $\begin{array}{l}\text { Actividad 1: Presentación esquema en tres etapas (en el embarazo, cuando el pequeño es } \\
\text { un bebé y cuando ambos son niños. } \\
\text { Actividad 2: Casos prácticos. }\end{array}$ \\
\hline $\begin{array}{l}\text { SESIÓN 8: "INDEPENDENCIA, } \\
\text { AUTONOMÍA Y SEGURIDAD" }\end{array}$ & $>$ & $\begin{array}{l}\text { Actividad 1: ¿Cómo fomentáis la autonomía? } \\
\text { Actividad 2: Presentación de ficha/pictogramas a realizar para potenciar la autonomía del } \\
\text { pequeño. }\end{array}$ \\
\hline $\begin{array}{l}\text { SESIÓN 9: } \\
\text { "CICLO DE CONDUCTAS } \\
\text { DESADAPTATIVAS" }\end{array}$ & $>$ & $\begin{array}{l}\text { Actividad 1: Repasar los pictogramas de la sesión anterior con nuevas posibles } \\
\text { necesidades. } \\
\text { Actividad 2: Presentación de casos prácticos: } \\
\text { Desde el punto de vista del niño ¿Por qué ha actuado así? } \\
\text { Desde el punto de vista de la mamá y papá ¿Por qué ha actuado así? }\end{array}$ \\
\hline $\begin{array}{c}\text { SESIÓN 10: } \\
\text { "IMPORTANCIA DE LA FAMILIA" }\end{array}$ & $>$ & $\begin{array}{l}\text { Actividad 1: Técnica del árbol } \\
\text { ¿Qué es ser buen padre?-> características positivas de los padres y madre } \\
\text { ¿Quién creéis que riega o alimenta promover esas características? } \\
\text { ¿Qué es ser buen hijo/a?-> características positivas de los niños/as } \\
\text { ¿Qué nos gustaría fomentar de nuestros hijos/as? }\end{array}$ \\
\hline $\begin{array}{c}\text { SESIÓN 11: } \\
\text { "IMPORTANCIA DEL JUEGO" }\end{array}$ & & $\begin{array}{l}\text { Actividad 1: Junglespeed. } \\
\text { Actividad 2: Evaluación del programa. }\end{array}$ \\
\hline
\end{tabular}

